\title{
Histological evaluation of bone biopsy results during PVP or PKP of vertebral compression fractures
}

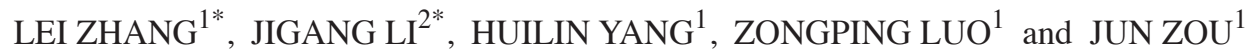 \\ ${ }^{1}$ Department of Orthopedic Surgery, The First Affiliated Hospital of Soochow University, Suzhou 215006; \\ ${ }^{2}$ Department of Orthopedic Surgery, The Central Hospital of Zibo, Zibo 255000, P.R. China
}

Received July 8, 2012; Accepted September 17, 2012

DOI: 10.3892/ol.2012.944

\begin{abstract}
Percutaneous vertebroplasty (PVP) or percutaneous kyphoplasty (PKP) is utilized for the treatment of vertebral compression fractures (VCFs). The present study was conducted to evaluate biopsy results obtained from VCFs during PVP or PKP. Between January 2003 and December 2011, 692 vertebral body biopsies were obtained from 546 patients (442 females and 104 males; mean age, 72.3 years), who underwent PVP or PKP for treatment of VCFs. A history of malignancy was identified in 44 patients. Histological/immunohistochemical evaluations were performed by experienced pathologists and $89.9 \%$ of biopsies obtained from 546 patients were suitable for histological/immunohistochemical evaluation. The biopsy results of 398 patients were in good agreement with the diagnosis of osteoporotic VCFs. Among the 44 patients with a history of malignancy, malignancy was identified in 25 patients, while osteoporosis was identified in 16 patients. Biopsies of the other 3 patients were not suitable for pathological examination. There were only 2 patients with previously undiagnosed malignancy. One patient was diagnosed with multiple myeloma, while another patient was diagnosed with metastatic carcinoma. In the present study, the rate of unsuspected malignancy was $0.4 \%$. No symptomatic complications occurred as a result of biopsy. The results indicate that biopsy is safely performed during PVP or PKP and it is an efficacious procedure that verifies the pathological process and assists in identifying previously unsuspected processes responsible for VCFs. We recommend obtaining the biopsy of every collapsed vertebral body during PVP or PKP procedures.
\end{abstract}

Correspondence to: Professor Huilin Yang or ProfessorZongping Luo, Department of Orthopedic Surgery, The First Affiliated Hospital of Soochow University, No. 188 Shizi Street, Suzhou 215006, P.R. China

E-mail: zlsuda2003@163.com

*Contributed equally

Key words: vertebral compression fractures, percutaneous vertebroplasty, percutaneous kyphoplasty, biopsy

\section{Introduction}

Vertebral compression fractures (VCFs) are the most common type of osteoporotic fracture (1) and affect over 200 million individuals worldwide $(2,3)$. Postmenopausal bone loss is one of the leading causes of VCFs. However, a variety of primary malignant conditions or secondary to non-skeletal primary neoplasms (multiple myeloma, lymphoma and metastatic disease) may also be responsible. Diagnosis of osteoporosis is based on clinical and radiological imaging observations, including DEXA examination. However, these processes often do not distinguish between neoplastic and osteoporotic etiology (4).

In previous years, minimally invasive techniques, including percutaneous vertebroplasty (PVP) and percutaneous kyphoplasty (PKP), have been developed. PVP and PKP procedures have been relatively effective in reducing fracture-related pain, diminishing disability and accelerating complete recovery of orthopedic injuries (5-8). Acquisition of a biopsy sample is performed during PVP or PKP procedures. Biopsy samples are also obtained from patients with normal laboratory reports. The outcome of bone biopsies with unexpected malignancy in cases of vertebral compression fracture treated with PVP or PKP have been previously reported (9-12). The purpose of the present study was to review the results of biopsies obtained during PVP or PKP. Specifically, we aimed to determine the incidence of previously undiagnosed malignancies in our own patient population. We also wanted to determine the efficacy of obtaining biopsy during PVP or PKP to estimate the effect of biopsy results on subsequent patient care and management.

\section{Patients and methods}

Patients. Between January 2003 and December 2011, a total of 546 patients were treated with PVP or PKP at the Department of Orthopedic Surgery of the First Affiliated Hospital of SooChow University, Suzhou, China. This included 104 males (19\%) and 442 females (81\%), with a mean age of 75.3 years (range, 56-93 years). In total, we treated 692 cases of VCFs. During PVP or PKP procedures, 692 vertebral body biopsies were obtained; 427 level 1, 95 level 2, 21 level 3 and 3 level 4 biopsies were obtained in this study. Biopsy levels included the following cases: T5 (3), T6 (8), T7 (14), T8 (23), T9 (33), 
T10 (30), T11 (62), T12 (149), L1 (189), L2 (85), L3 (50), L4 (31) and L5 (15). Prior to surgery, 502 patients (92\%) had no history of malignancy and 44 patients (8\%) had a history of malignancy, which was considered to be the cause of their compression fractures. The group of patients with known history of malignancy was comprised of patients with carcinoma of the lung (13), breast (7), multiple-myeloma (6), liver (5), bladder (4), prostate (4), pancreatic gland (2), chromatophoroma (2) and gastric cancer (1).

Obtainment of biopsies. During the PVP and PKP procedures, 122 and 570 biopsies were obtained, respectively. The patients were kept in the prone position for every procedure. In all cases, the transpedicular approach was used to access the collapsed vertebral body. For this purpose, we used biplane fluoroscopy with two C-arms statically placed in the posteroanterior and lateral positions. A 3-mm diameter trephine (Medtronic, Minneapolis, MN, USA) was passed immediately into the vertebral body through the previously inserted working cannula. The working cannula was inserted prior to infusion of the biological cement or prior to insertion of the inflatable bone tamp in the PVP and PKP procedures, respectively. Biopsies were sent for pathological examination immediately following the completion of the procedure and were fixed in $10 \%$ neutral-buffered formalin. The decalcified biopsy material was embedded in paraffin, sectioned and stained with hematoxylin and eosin. The stained sections were examined by a pathologist under a light microscope. If malignancy was identified, the biopsy was reviewed by a senior pathologist.

The present study was approved by the institutional review board (IRB) of The First Affiliated Hospital of SooChow University, Suzhou, China and informed consent was obtained from each patient or candidate.

\section{Results}

Following the surgical procedure, all patients recovered from the VCF and reported rapid pain relief. Symptomatic complications caused by PVP or PKP were not observed. All patient charts were available for review and $89.9 \%$ of biopsies obtained from 546 patients were suitable for histological evaluation. The biopsy results of 448 patients were consistent with the diagnosis of osteoporotic VCFs (OVCFs). In the majority of biopsy specimens, trabecular bone with erratic levels of fibrosis and granulation tissue reflected various stages of progressive fracture healing. Among the 44 patients with an initial malignant condition, malignancy was identified as the cause of compression fractures in 25 patients. In the remaining 19 patients with malignancy, the suspected etiology behind the VCFs was not confirmed. In this case, osteoporosis was identified in 16 patients, while the biopsies of the remaining 3 patients were not suitable for pathological examination. We observed 2 patients whose malignancy was not suspected through pre-operative imaging or clinical symptoms. One patient (male, 68 years old) suffered from multiple myeloma (Fig. 1), while another patient (female, 62 years old) suffered from metastatic carcinoma (Fig. 2). In the present study, the rate of unsuspected malignancy was $0.4 \%$. These two patients were subsequently transferred to

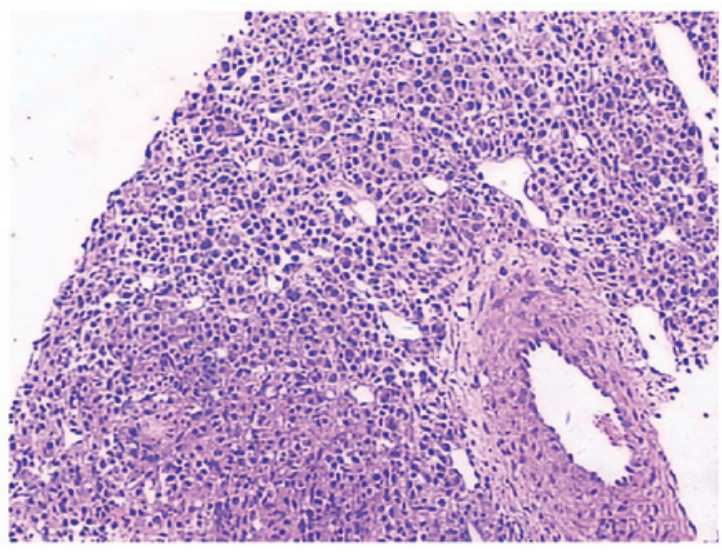

Figure 1. Photomicrograph stained with hematoxylin and eosin (original magnification x100), demonstrating diffuse collections of multiple myeloma cells. Biopsy of a 68-year old male with compression fracture at T12 and no prior history of cancer.

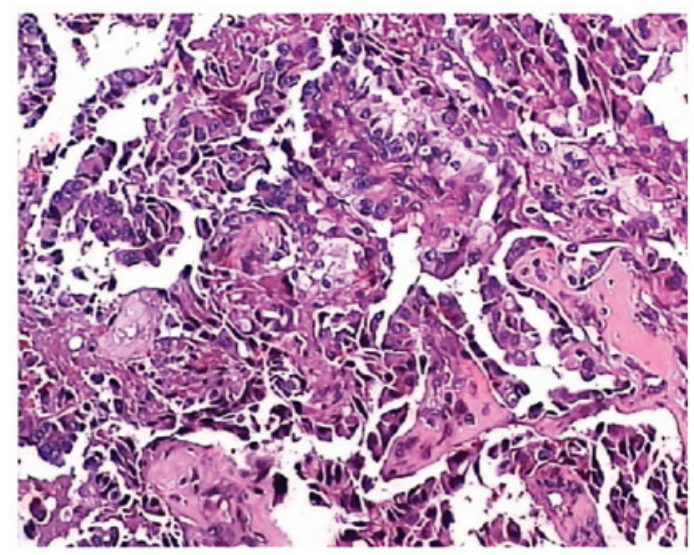

Figure 2. Photomicrograph stained with haematoxylin and eosin (original magnification x100), reveals adenocarcinomatous nests demonstrating markedly typical malignant cells that are consistent with metastatic carcinoma. Biopsy of a 62-year old female with compression fracture at L3 and no previous history of cancer.

the oncology department for further diagnostic tests and treatment.

\section{Discussion}

Prior to PVP or PKP procedures, diagnosis of OVCFs was based on clinical and radiological observations, including X-ray, CT, MRI, bone material density and ECT. However, in certain cases these pre-operative investigations fail to provide correct diagnosis of the condition $(4,10,11)$. It has been reported that the rate of distinguishing benign and malignant compression fractures of the spine through MRI is between 55 and 94\% (13-15). Therefore, patients with malignancy require accurate pathological diagnosis to select the correct program for further treatment of their condition. Following administration of steroids, chemotherapeutic drugs or radiotherapy, patients with lymphoma, multiple myeloma or metastatic carcinomas are prone to VCFs owing to bone loss. In addition, the majority of patients are elderly. However, osteoporosis is often assumed to be the natural cause of VCFs and aging increases 
occurrence of OVCFs (10). Malignancy and osteoporosis was observed in the same patient. In the majority of cases, osteoporosis is considered to be the correct assumption, however a misdiagnosis may have a disastrous prognosis. The nature of the underlying pathology associated with vertebral collapse is important in correct prognosis. Moreover, it is also required for assessing a patient's response to therapy and long-term patient care (10). The nature of the underlying pathology associated with vertebral collapse is also important for VCF patients with a medical history of malignancy, wherein the primary tumor is not involved with the spine.

In the case of patients with a history of malignancy or suspected malignancy, the outcomes of bone biopsies obtained during PVP or PKP have been inconsistent, as the percentage of malignancy diagnosis varies between 32.4 and $89 \%(16,17)$. In the present study, 25 patients $(56.8 \%)$ were diagnosed with malignancy through biopsy samples obtained during PVP or PKP. These patients had a history of malignancy or suspected malignancy and were transferred to the oncology department for specialized treatment. Medical history of malignancy was reported in 16 patients, however, their biopsy samples did not provide any evidence of malignancy. Therefore, osteoporosis was the cause of VCFs in these patients and the 16 patients underwent anti-osteoporosis treatment.

There are instances where biopsy provides evidence of malignancy when the patient has no clinical or imaging evidence (9-12). Togawa et al (10) demonstrated that biopsy provided a definitive diagnosis in one case of unsuspected multiple myeloma in their population $(0.7 \%$ of all biopsies). Muijs et al (9) observed malignancy evidence in the biopsies of 3 patients ( $3.8 \%$ of all biopsies), whose malignancies were previously undiagnosed. This included 2 patients with multiple myeloma stage IIa and 1 patient with chondrosarcoma grade I. These biopsy samples were obtained during PVP, which were conducted to treat OVCFs in these patients. Shindle et al (11) reported that $3 / 238(1.3 \%)$ patients were diagnosed with unsuspected lymphoma during PKP, through analysis of biopsy samples obtained during the treatment. The authors obtained a total of 423 biopsy samples during diagnosis of 238 patients. More recently, Schoenfeld et al (12) reported that malignancy was observed in 4 patients through analysis of biopsy samples obtained during PVP or PKP. In this case, the malignancy was detected in the previous diagnosis of 3 patients and 2 patients $(0.4 \%)$ were diagnosed with malignancy by biopsy during PKP. The malignancy was not suspected in these cases, as these patients revealed normal pre-operative imaging and did not exhibit any clinical symptoms. In summary, vertebrae biopsy is highly recommended in such cases and not only clarifies the nature of disease but also contributes to treatment development in VCF patients with a history of malignancy. For patients who do not demonstrate clinical malignant evidence, vertebral biopsy is an important method for confirmation of the malignancy diagnosis.

According to clinical and radiological observations of VCFs, biopsy results were consistent with the diagnosis of OVCFs in 398 patients included in the present study. In the majority of biopsy specimens, trabecular bone with fibrosis and granulation tissue reflected the various stages of progressive fracture healing. Diamond et al (18) characterized the histological process of fracture healing in cancellous bone of vertebral body and divided this process into four stages. Stage 1 included necrosis and granulation tissue; stage 2 included chondrogenesis and bone matrix synthesis; stage 3 included endochondral ossification and woven bone formation; and stage 4 included bone remodeling and modeling. The present findings revealed that the majority of fractures were early stage and may be attributed to the early procedure time and biopsy sample size.

The posterolateral approach is normally used for obtaining biopsy under fluoroscopic or CT guidance in cases of thoracic or lumbar spine (19-21). However, the risk correlated with this process varies from 0 to $26 \%$ and is associated with various complications, including pneumothorax, hematoma, neurological lesions, root pain and infective disorders. Posterolateral biopsy is also correlated with the risk of tumor metastasis by needle sheath in malignancy (22). In order to avoid these complications, the biopsy was previously performed using a transpedicular approach under CT or fluoroscopic guidance (22,23). During PVP or PKP in the present study, a cannulated trocar was inserted into the vertebral body through a transpedicular approach. Following removal of the guide wire, a trephine was used to obtain the biopsy sample of the vertebral body. The procedures provided a definite path to acquire a biopsy sample and did not significantly increase surgical time. In the case of malignant patients with VCFs, we completed the diagnosis and surgery during PKP or PVP.

During PVP or PKP procedures, the time and frequency of biopsy material acquisition remains debated. Togawa et al and Schoenfeld et al $(10,12)$ advise that biopsy should be performed while conducting the first vertebral augmentation procedure. However, Allen et al (24) suggested that patients undergoing first-time vertebral augmentation should be considered for vertebral biopsy. The present study and additional studies $(4,10,12)$ have confirmed malignant biopsy results in patients, who did not exhibit any other clinical malignant evidence. However, the rate of detection was low in such cases. Moreover, a biopsy obtained during PVP or PKP does not increase morbidity in the patients and the biopsy aids identification of the underlying pathology. Early detection of malignant disease is more important than the risks associated with biopsy as effective timely treatment for malignancy is unlikely to be provided to patients who do not recieve early diagnosis through this process. Considering the potential advantages of biopsy during PVP or PKP, we suggest that biopsy of every collapsed vertebra should be performed during every procedure of PVP or PKP, even in patients with no history of malignancy or suspected malignancy.

Previously, a meta-analysis of percutaneous spine biopsy demonstrated that the rates of adequacy ( $92.6 \mathrm{vs} .90 .1 \%)$ and accuracy ( 90.2 vs. $88.1 \%$ ) were slightly higher than those of fluoroscopy. However, these increases were not significant (25). A previous study reported that diagnostic accuracy of percutaneous spine biopsy is more than $90 \%$ in cases of known or suspected malignancy (26). Therefore, researchers must focus on improving the efficiency of needle aspiration biopsy to obtain sufficient pathological cells. Kattapuram et al (27) reported that the accuracy achieved by large needles was slightly higher than that of fine. It has been suggested that needles with a diameter of at least $2 \mathrm{~mm}$ are suitable for obtaining samples sufficient for histological evalu- 
ation $(20,28,29)$. Ward et al (29) demonstrated that $3.5 \mathrm{~mm}$ trephine were suitable for obtaining samples. Larger diameter needles were more suitable for obtaining adequate bone cores for histological evaluation. However, during PVP or PKP, the diameter of biopsy needles is limited by the working cannula. The biopsy and infusion of bone cement are performed under cannula, which is first inserted into the pedicle. Therefore, the biopsy procedure is not associated with the risks of inflicting injuries to vital tissues, including major vessels and nerve roots of spinal cord. While obtaining patient biopsies during PKP or PVP, we did not observe any complications. Therefore, we recommend using biopsy needles with maximal diameter in order to obtain more bone cores. In the present study, a biopsy needle with a diameter of $3 \mathrm{~mm}$ was utilized safely and effectively during the PVP or PKP procedures.

In conclusion, PVP and PKP are effective treatments for VCFs. If biopsy samples are obtained while performing these procedures, no increase in morbidity or surgical duration is observed. Biopsy aids identification of the underlying pathology. In the present study, the rate of unsuspected malignance was $0.4 \%$. Obtaining a biopsy sample is relatively safe and easy, therefore we recommend collapsed vertebral body biopsy during every PVP or PKP procedure. In addition we recommend using a biopsy needle of maximal diameter for under cannula. For effective VCF management, it is important to obtain a biopsy sample during PVP or PKP procedure.

\section{References}

1. Ross PD: Clinical consequences of vertebral fractures. Am J Med 103: 30S-42S, 1997.

2. Garfin SR, Buckley RA and Ledlie J; Balloon Kyphoplasty Outcomes Group: Balloon kyphoplasty for symptomatic vertebral body compression fractures results in rapid, significant and sustained improvements in back pain, function and quality of life for elderly patients. Spine (Phila Pa 1976) 31: 2213-2220, 2006.

3. van Schoor N, Smit J, Twisk JW and Lips P: Impact of vertebral deformities, osteoarthritis and other chronic diseases on quality of life: a population based study. Osteoporos Int 16: 749-756, 2005.

4. Ho CS, Choi WM, Chen CY, Chen WY and Chan WP: Metastasis in vertebra mimicking acute compression fractures in a patient with osteoporosis: MRI findings. Clin Imaging 29: 64-67, 2005.

5. Ledlie JT and Renfro M: Balloon kyphoplasty: one year outcomes in vertebral body height restoration, chronic pain and activitiy levels. J Neurosurg 98: 36-42, 2003.

6. Crandall D, Slaughter D, Hankins PJ, Moore C and Jerman J: Acute versus chronic vertebral compression fractures treated with kyphoplasty: early results. Spine J 4: 418-24, 2004.

7. Evans AJ, Jensen ME, Kip KE, et al: Vertebral compression fractures: pain reduction and improvement in functional mobility after percutaneous polymethylmethacrylate vertebroplasty retrospective report of 245 cases. Radiology 226: 366-372, 2003.

8. Mathis JM, Ortiz AO and Zoarski GH: Vertebroplasty versus kyphoplasty: a comparison and contrast. AJNR Am J Neuroradiol 25: 840-845, 2004.

9. Muijs SP, Akkermans PA, van Erkel AR and Dijkstra SD: The value of routinely performing a bone biopsy during percutaneous vertebroplasty in treatment of osteoporotic vertebral compression fractures. Spine (Phila Pa 1976) 34: 2395-2399, 2009.
10. Togawa D, Lieberman IH, Bauer TW, Reinhradt MK and Kayanja MM: Histological evaluation of biopsies obtained from vertebral compression fractures: unsuspected myeloma and osteomalacia. Spine (Phila Pa 1976) 30: 781-786, 2005.

11. Shindle MK, Tyler W, Edobor-Osula F, Gradner MJ, Shindle L, Toro J and Lane JM: Unsuspected lymphoma diagnosed with use of biopsy during kyphoplasty. J Bone Joint Surg Am 88: 2721-2724, 2006.

12. Schoenfeld AJ, Dinicola NJ, Ehrler DM, et al: Retrospective review of biopsy results following percutaneous fixation of vertebral compression fractures. Injury 39: 327-333, 2008.

13. An HS, Andreshak TG, Nguyen C, Williams A and Daniels D: Can we distinguish between benign versus malignant compression fractures of the spine by magnetic resonance imaging? Spine (Phila Pa 1976) 20: 1776-1782, 1995.

14. Rupp RE, Ebraheim NA and Coombs RJ: Magnetic resonance imaging differentiation of compression spine fractures or vertebral lesions caused by osteoporosis or tumor. Spine (Phila Pa 1976) 20: 2499-2504, 1995.

15. Moulopoulos LA, Yoshimitsu K, Johnston D, Leeds NE and Libshitz HI: MR prediction of benign and malignant vertebral compression fractures. J Magn Reson Imaging 4: 667-674, 1996.

16. Minart D, Vallee JN, Cormier E and Chiras J: Percutaneous coaxial transpedicular biopsy of vertebral body lesions during vertebroplasty. Neuroradiology 43: 409-412, 2001.

17. Erie L, Mark HB, Leszek P, et al: Percutaneous CT-guided biopsy of osseous lesion of the spine in patients with known or suspected malignancy. AJNR Am J Neuroradiol 25: 1583-1588, 2004.

18. Diamond TH, Clark WA and Kumar SV: Histomorphometric analysis of fracture healing cascade in acute osteoporotic vertebral body fractures. Bone 40: 775-780, 2007.

19. Bender CE, Berquist TH and Wold LE: Imaging-assisted percutaneous biopsy of the thoracic spine. Mayo Clin Proc 61: 942-950, 1986.

20. Fyfe IS, Henry AP and Mulholland RC: Closed vertebral biopsy. J Bone Joint Surg Br 65: 140-143, 1983.

21. Laredo JD and Bard M: Thoracic spine: percutaneous trephine biopsy. Radiology 160: 485-489, 1986.

22. Jelinek JS, Kransdorf MJ, Gray R, Aboulafia AJ and Malawer MM: Percutaneous transpedicular biopsy of vertebral body lesions. Spine (Phila Pa 1976) 21: 2035-2040, 1996.

23. Pierot L and Boulin A: Percutaneous biopsy of the thoracic and lumbar spine: transpedicular approach under fluoroscopic guidance. AJNR Am J Neuroradiol 20: 23-25, 1999.

24. Allen RT, Kum JB, Weidner N, Hulst JB and Garfin SR: Biopsy of osteoporotic vertebral compression fractures during kyphoplasty: unsuspected histologic findings of chronic osteitis without clinical evidence of osteomyelitis. Spine (Phila Pa 1976) 34: 1486-1491, 2009.

25. Nourbakhsh A, Grady JJ and Garges KJ: Percutaneous spine biopsy: a meta-analysis. J Bone Joint Surg Am 90: 1722-1725, 2008.

26. Lis E, Bilsky MH, Pisinski L, Boland P, Healy JH, O'malley B and Krol G: Percutaneous CT-guided biopsy of osseous lesion of the spine in patients with known or suspected malignancy. AJNR Am J Neuroradiol 25: 1583-1588, 2004.

27. Kattapuram SV, Khurana JS and Rosenthal DI: Percutaneous needle biopsy of the spine. Spine (Phila Pa 1976) 17: 561-564, 1992.

28. Akerman M, Berg NO and Persson BM: Fine needle aspiration biopsy in the evaluation of tumor-like lesions of bone. Acta Orthop Scand 47: 129-136, 1976.

29. Ward JC, Jeanneret B, Oehlsehlegel C and Magerl F: The value of percutaneous transpedicular vertebral bone biopsies for histologic examination. Results of an experimental histopathologic study comparing two biopsy needles. Spine (Phila Pa 1976) 21: 2484-2490, 1996 